\title{
Development and Validation of Stability Indicating RP-HPLC Method for Rivaroxaban and Its Impurities
}

\author{
Yashpalsinh N Girase ${ }^{1}$, Srinivasrao $\mathrm{V}^{2}$, DiptiSoni ${ }^{3}$ \\ ${ }^{1}$ Research Scholar, Pacific Academy of higher Education and Research University, Udaipur, India \\ ${ }^{2}$ Department of Research and Development, Pacific University, Udaipur, India \\ ${ }^{2,3}$ Department of Chemistry, Pacific Academy of higher Education and Research University, Udaipur, India
}

Received: 7 August, 2017; Accepted: 18 February, 2017; Published: 5 March, 2018

*Corresponding author: Srinivasrao V, Department of Chemistry, Department of Research and Development, Macleods Pharma Ltd, Udaipur, India, E-mail: drvsraoemail@gmail.com

\begin{abstract}
Rivaroxaban is oxazolidinone derivative having anticoagulant activity. In literature few analytical methods are discuss about estimation of rivaroxaban; but rarer discussion is available for rivaroxaban impurity profile. The objective of this study is to develop and validate RP-HPLC method for the qualitative analysis of Rivaroxaban. The chromatographic separation was achieved on ZorbaxSB C18 (250 mm X 4.6 mm, $3.5 \mu$ ) HPLC column using buffer (0.02M mono basic potassium di hydrogen phosphate) and solvent mixture (acetonitrile: methanol mixture) ingradient programme. The developed methods were validated as per ICH guideline and found to be specific, precise, sensitive and robust.
\end{abstract}

Keywords: Rivaroxaban; oxazolidinone derivative; Anticoagulant drug, RP-HPLC method; related substance; impurity profile;

\section{Introduction}

Rivaroxabanis 5 - chloro-N-(\{(5S)-2-oxo-3-[4- (3oxomorpholin-4-yl)phenyl]-1,3-oxazolidin-5yl\}methyl) thiophene-2-carbinoxamide Figure 1.

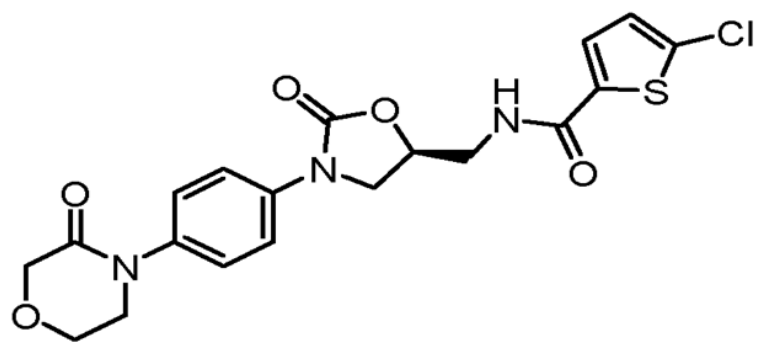

Figure 1: Rivaroxaban

Molecular mass: 435.88

Molecular formula: $\mathrm{C}_{19} \mathrm{H}_{18} \mathrm{ClN}_{3} \mathrm{O}_{5} \mathrm{~S}$

In November 2008 the Therapeutic Goods Administration approved new oral anticoagulant drug Rivaroxaban for the prevention of venous thrombosis in patients having elective knee or hip replacement [1, 2]. Rivaroxaban is an oxazolidinone derivative anticoagulant that competitive reversible antagonist of activated factor X (Xa). Factor Xa is the active component of the prothrombinase complex that catalyses conversion of prothrombin (factor II) to thrombin (factor IIa). It is a highly selective direct Factor Xa inhibitor with oral bioavailability and rapid onset of action. Rivaroxabin does not inhibit thrombin (activated Factor II), and no effect on platelets have been demonstrated.

There is no official monograph available for Rivaroxaban or drug product in any pharmacopiea. A preliminary survey of literature for suitable method development for Rivaroxaban has been made $[3,4]$. Review of literature suggests that no extensive work has been carried out for the routine analysis of Rivaroxaban, Which can address all process impurities and degradation profiles [5-7]. Monitoring of impurity profiling is very important for quality of drug and patient safety purpose. Also literature survey shows few analytical methods were published for the estimation of Rivaroxaban during formulation and bio availability study for the assay purpose. But rare discussion is available for Rivaroxaban impurity profile study. This study shows detail discussion on monitoring of commercial rout of synthesis and impurity profiling Figure 2, 3. Hence the aim of the present work was to develop accurate and robust routine HPLC method.

\section{Experimental}

\section{Material and Reagents}

Pure Rivaroxaban was obtained using commercial route of synthesis as per the process described in the Figure-2 [9]. The related impurities including process impurities and degradant impurities (as described in Figure-3) were synthesised in house. The rivaroxaban standard and impurities were characterized using proton nuclear magnetic resonance and mass spectrometry equipped with HPLC. HPLC grade acetonitrile, methanol was procured from J T Baker. Analytical grade potassium dihydrogen phosphate and orthophosphoric acid obtained from Merck chemicals. HPLC grade water obtained from Millipore system was used throughout the analysis. Ion pair reagent Octane sulfonic acid was purchased of Ranchem make. 


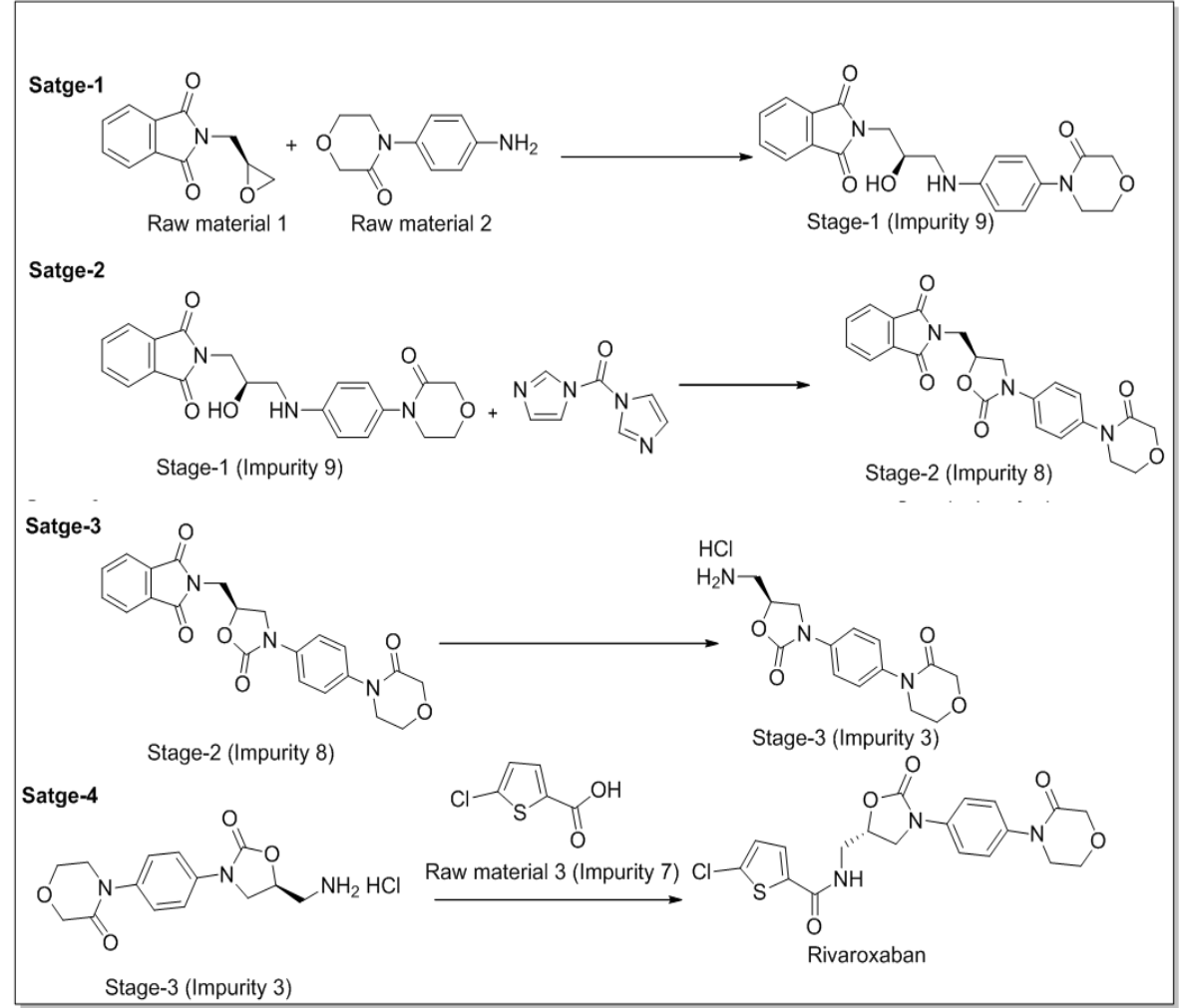

Figure 2: Commercial route of synthesis for rivaroxaban

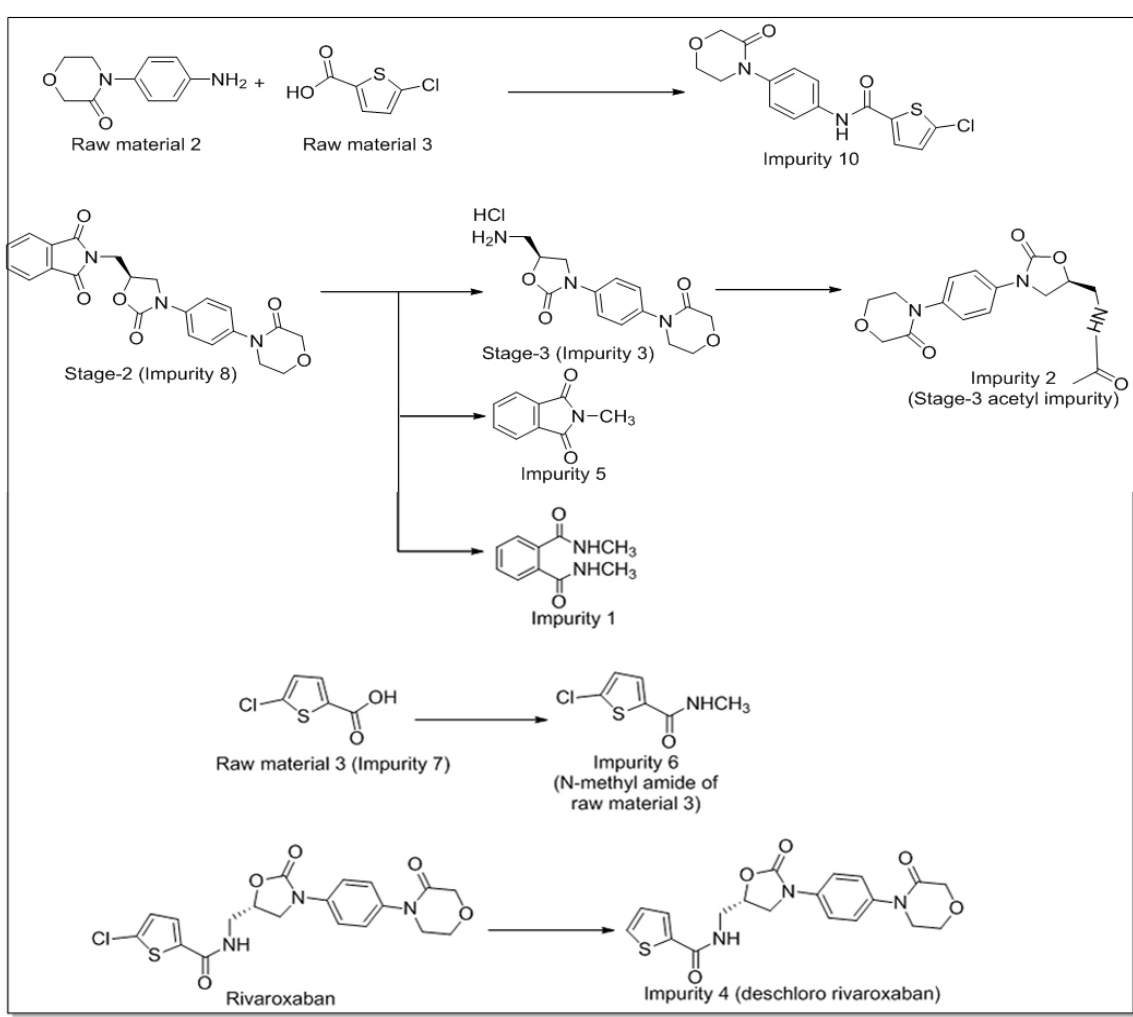

Figure 3: Impurity profiling of rivaroxaban 


\section{RP-HPLC Method}

\section{Optimization Experiments}

In the process of developing RPHPLC method three key parameters were studied which influence the selectivity such as chemistry of stationery phase, $\mathrm{pH}$ of the buffer, and organic modifiers. Phophate buffer with Octane sulphonicacid, $\mathrm{pH}$ was screened as pH3.0,4.0 and 5.0. HPLC columns were used for development of method were Inertsil ODS 3V, Zorbax phenyl, Kromasil C18 and ZorbaxSBC18. Methanol and Acetonitrile were chosen individual and in different ratio as organic modifier. The impurities spiked solution in Rivaroxaban and sample of all stressed condition were studied and recorded and method was optimized with satisfactory resolutions among all impurities on ZorbaxSBC18 column in Figure 4.

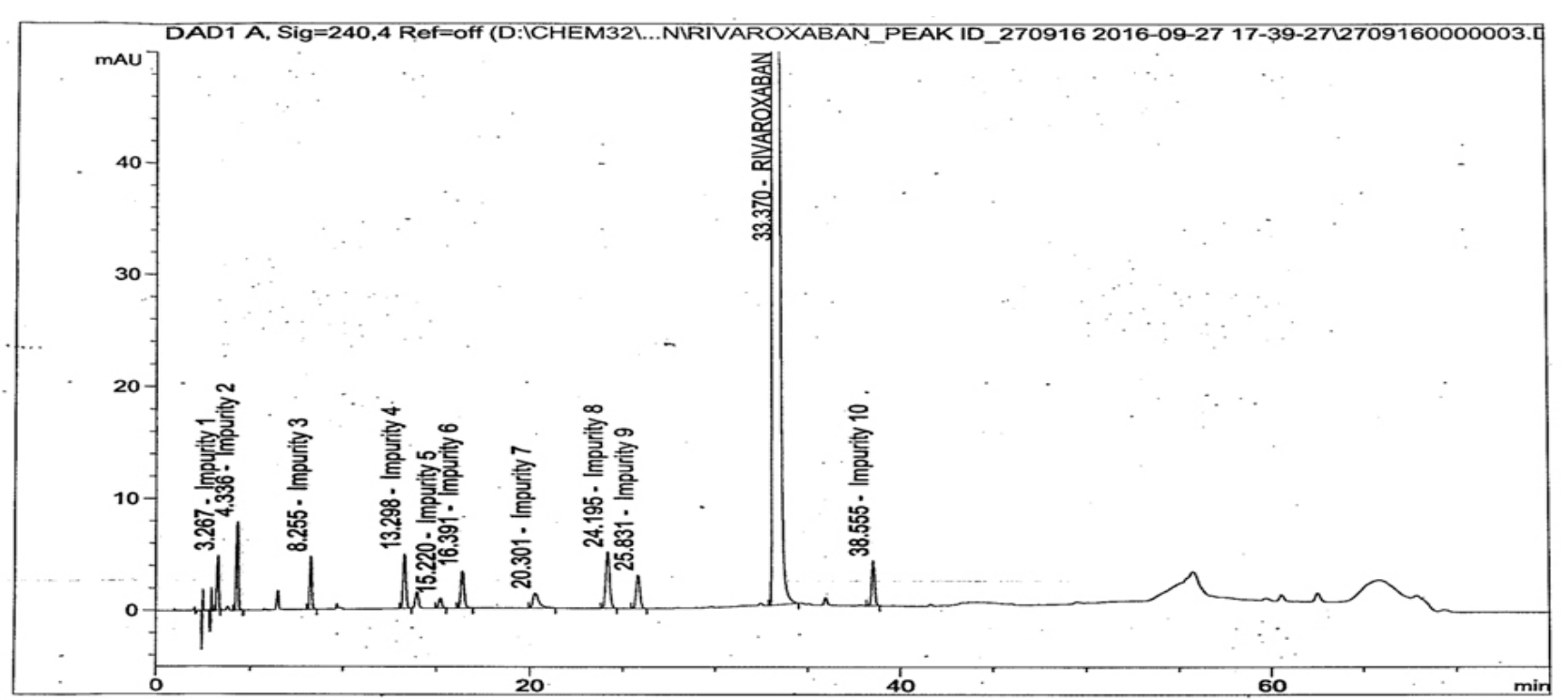

Figure 4: All impurities spiked at 0.15\%level in Rivaroxaban

\section{Instrumentation and Chromatographic Conditions}

Agilent HPLC 1200 (Agilent Technologies, Germany) equipped with photodiode array detector was used for method development, forced degradation studies and method validation. Zorbax SB C18 (250mmX4.6 mm $3.5 \mu$ ) HPLC column. Column thermostat at $45^{\circ} \mathrm{C}$ was used for the impurities separation. Buffer was prepared using $0.02 \mathrm{M}$ of anhydrous potassium dihydrogen phosphate and $1 \mathrm{gm}$ of Octane sulphonic acid solution was adjusted to $\mathrm{pH} 3.0$ with orthophosphoricacid. Solvent mixture was prepared Acetonitrile: Methanol in ratio $820: 180 v / v$. Mobile phase A was prepared by mixing Buffer and solvent mixture in ratio $800: 200 \mathrm{v} / \mathrm{v}$. Mobile phase-B was prepared by mixing Buffer and solvent mixture in ratio of 200:800 v/v. The flow rate and injection volumes were $1.0 \mathrm{ml} /$ min and $10 \mu$ lrespectively. The analysis was carried out under the gradient condition as time $(\mathrm{min}) / \mathrm{A}(\mathrm{v} / \mathrm{v}): \mathrm{B}(\mathrm{v} / \mathrm{v}) ; \mathrm{T}_{0.01} / 85: 15$, $\mathrm{T}_{22.0} / 75: 25, \mathrm{~T}_{35.0} / 55: 45, \mathrm{~T}_{50.0} / 40: 60, \mathrm{~T} 65.0 / 40: 60, \mathrm{~T}_{66.0} / 85: 15$ and $\mathrm{T}_{75.0} / 85: 15$. The data was acquired at $240 \mathrm{~nm}$ for Impurity 1, to Impurity 10; Run time kept 75 min. Chromatographic data processed by using chemstation and chromline HPLC software. The photodiode array detector was used to determine the peak purity of stressed sample.

Preparation of Solutions and Analytical Procedure (System Suitability)
Diluent was prepared by mixing solution A and water in the ratio of 500:500 v/v. Solution A was prepared by mixing methanol and Acetonitrile in the ratio of 500:500 v/v. The test sample solution having concentration of $1000 \mu \mathrm{g} / \mathrm{ml}$ was prepared for the determination of related substances. The stock solution was prepared by dissolving each impurity (Impurity1, Impurity2, Impurity3, Impurity4, Impurity5, Impurity6, Impurity7, Impurity8, Impurity9 and Impurity10) at concentration about $15 \mu \mathrm{g} / \mathrm{mL}$ in diluent and further diluted up to $1.5 \mu \mathrm{g} / \mathrm{ml}$ along with Rivaroxaban standard at $1000 \mu \mathrm{g} / \mathrm{ml}$ to prepare the system suitable solution. Inject diluted standard solution in six replicates into the chromatograph and record the chromatogram\% RSD for area response of Rivaroxaban peak from six replicate injections of diluted standard solution should not be more than 5.0. The blank, system suitability solution and sample solution of $1000 \mu \mathrm{g} / \mathrm{ml}$, were injected separately and chromatographed under the optimized chromatographic conditions. The resolution NLT 2.0, between Impurity 8peak and Impurity 9 were set as system suitability criteria. All impurities were quantified against $0.1 \%$ rivaroxaban diluted standard solution applying the derived Relative Response Factor (RRF). The relative retention time with respect to Rivaroxaban peak and RRF of all impurities are as shown in Table-1, 2. 


\begin{tabular}{|c|c|c|c|c|}
\hline \multicolumn{5}{|l|}{ Table-1: } \\
\hline $\begin{array}{c}\text { System } \\
\text { suitability } \\
\text { results }\end{array}$ & $\begin{array}{c}\text { Retention } \\
\text { Time of } \\
\text { Rivaroxaban } \\
\text { (minutes) }\end{array}$ & $\begin{array}{l}\text { Resolution } \\
\text { between } \\
\text { Imp-8 and } \\
\text { Imp-9 peak }\end{array}$ & Mean Area & $\begin{array}{c}\% \\
\text { RSD }\end{array}$ \\
\hline $\begin{array}{c}\text { Unaltered } \\
\text { (Mean } \\
\text { Repeatability) }\end{array}$ & 34.529 & 3.87 & 27411.35167 & 0.40 \\
\hline
\end{tabular}

Table-2: Rivaroxaban and its impurities elution order and relative response factor

\begin{tabular}{|c|c|c|c|}
\hline S. No & Name & RRT & RRF \\
\hline 1 & Impurity-1 & 0.1 & 0.7 \\
\hline 2 & Impurity-2 & 0.13 & 0.74 \\
\hline 3 & Impurity-3 & 0.26 & 1 \\
\hline 4 & Impurity-4 & 0.39 & 1.06 \\
\hline 5 & Impurity-5 & 0.45 & 1.53 \\
\hline 6 & Impurity-6 & 0.48 & 0.66 \\
\hline 7 & Impurity-7 & 0.58 & 0.57 \\
\hline 8 & Impurity-8 & 0.72 & 1.25 \\
\hline 9 & Impurity-9 & 0.77 & 1.05 \\
\hline 10 & Rivaroxaban & 1 & 1 \\
\hline 11 & Impurity-10 & 1.16 & 0.57 \\
\hline
\end{tabular}

\section{Validation}

\section{Specificity (Selectivity)}

Specificity is the ability of method to measure the analyte in presence of its potential impurities. Stress testing of the drug substance performed to identify likely degradation impurities, which intern help to establish the degradation pathways and intrinsic stability of the molecule and validate the stabilityindicating power of the analytical procedures used $[8,9]$.

The specificity of developed RP-HPLC method for Rivaroxaban was determined in presence of its impurities (Impurity 1 to Impurity 10 ) and degradation products. Forced degradation studies were also performed on Rivaroxaban to provide an indication of the stability-indicating property and specificity of the proposed method. The dry degradation study was performed by exposing the sample to different stress conditions such as light $\left(1.2\right.$ million lux hours), heat $\left(80^{\circ} \mathrm{C}\right.$ for 12 hours), hydrolytic condition $\left(45^{\circ} \mathrm{C}, 75 \% \mathrm{RH}\right.$ for $48 \mathrm{Hrs}$.). Wet degradation was performed as acid hydrolysis $(1 \mathrm{M} \mathrm{HCl}$ for $4 \mathrm{hrs}$ at $\left.80^{\circ} \mathrm{C}\right)$, base hydrolysis $\left(1 \mathrm{M} \mathrm{NaOH}\right.$ for $4 \mathrm{hrs}$ at $\left.80^{\circ} \mathrm{C}\right)$ and oxidation $\left(5 \% \mathrm{v} / \mathrm{v} \mathrm{H}_{2} \mathrm{O}_{2}\right.$ for $4 \mathrm{hrs}$ at $\left.40^{\circ} \mathrm{C}\right)$. Rivaroxaban was found to degrade significantly in Acid condition, but impurities are found well separated and found method is specific. Mass balance was observed during degradation for all the stressed samples.

\section{Linearity}

Linearity solutions were prepared by quantitative dilutions of the stock solution of impurity standard and main drug standard to obtain solutions at LOQ to $250 \%$ of the specification limited. Known impurity at $0.15 \%$ level and unknown impurity at $0.1 \%$ level. A series of solutions were prepared by quantitative dilutions of the stock solution of main drug to obtain solutions at $80 \%$ to $120 \%$ of the sample concentration.

Each solution was injected and areas were recorded. The linearity of peak areas versus different concentrations was evaluated for Rivaroxaban and its related impurities. The linear regression data for all the impurities plotted and correlation coefficient for all impurities was above 0.99.Linearity results are shown in Table 3.

Table-3: Linearity results

\begin{tabular}{|c|c|c|c|c|c|c|c|c|c|c|c|}
\hline $\begin{array}{c}\text { Validation } \\
\text { parameter }\end{array}$ & Imp-1 & Imp-2 & Imp-3 & Imp-4 & Imp-5 & Imp-6 & Imp-7 & Imp-8 & Imp-9 & API & Imp-10 \\
\hline Slope & 19622.48 & 27967.39 & 27129.84 & 32929.07 & 42741.57 & 20551.2 & 18186.55 & 35780.23 & 30839.05 & 28544.57 & 16287.76 \\
\hline Intercept & 220.3 & 440.56 & 420.31 & 539.82 & 1106.65 & 459.7 & 146.05 & 648.37 & 130.27 & 466.41 & 231.29 \\
\hline $\begin{array}{c}\text { Correlation } \\
\text { coefficient }\end{array}$ & 1 & 1 & 1 & 1 & 1 & 1 & 1 & 1 & 1 & 1 & 1 \\
\hline
\end{tabular}

\section{Limits of Detection and Quantification (LOD and LOQ)}

According to ICH Q2 (R1) recommendations the Limits Of Detection (LOD) and the Limit Of Quantification (LOQ) for Rivaroxaban and its process related impurities (Impurity 1 to Impurity 10 ) were estimated by calibration curve method [standard deviation of the response $(\sigma)$ and the slope $(S)]$, by injecting the series of dilute solutions of known concentration. The values of LOD and LOQ found are as depicted in Table-4.

Precision was studied at the LOQ level by injecting six individual preparations of Rivaroxaban and its impurities, followed by the calculation of \% RSD of the peaks areas. The $\%$ RSD of LOQ precision was below $10 \%$. 


\begin{tabular}{|c|c|c|c|}
\hline \multicolumn{4}{|c|}{ Table-4: LOQ and LOD values for Rivaroxaban and its impurity } \\
\hline S. No & Name & LOQ & LOD \\
\hline 1 & Impurity-1 & 0.033 & 0.019 \\
\hline 2 & Impurity-2 & 0.025 & 0.012 \\
\hline 3 & Impurity-3 & 0.026 & 0.011 \\
\hline 4 & Impurity-4 & 0.021 & 0.006 \\
\hline 5 & Impurity-5 & 0.037 & 0.015 \\
\hline 6 & Impurity-6 & 0.043 & 0.012 \\
\hline 7 & Impurity-7 & 0.048 & 0.021 \\
\hline 8 & Impurity-8 & 0.027 & 0.009 \\
\hline 9 & Impurity-9 & 0.034 & 0.015 \\
\hline 10 & Rivaroxaban & 0.03 & 0.018 \\
\hline 11 & Impurity-10 & 0.037 & 0.022 \\
\hline
\end{tabular}

\section{Precision}

The precision of method is degree of agreement between the results. Precision of the method was studied for system precision, method precision and intermediate precision. A standard solution of Rivaroxaban at $0.1 \%$ was injected for six time to determine the system precision of the method and \%RSD was calculated for Rivaroxaban. The \%RSD of system precision was found about $0.69 \%$.

Six separate test sample solutions of Rivaroxaban were prepared by spiking the related impurities (Impurity 1 to Impurity 10 ) at limit level (i.e.0.15\% for known and $0.1 \%$ for unknown). The \% RSD $(n=6)$ for each related impurities was evaluated and found in between $0.72 \%$ to $2.44 \%$.The similar procedure of method precision was carried out by a different analyst, using different mobile phase and diluent preparations and instrument on a different day with different lot of same brand column for intermediate precision study. The \%RSD of results for intermediate precision study was calculated and compared with the method precision results.

\section{Accuracy (Recovery)}

Accuracy of the method for all the impurities was determined by analyzing Rivaroxaban sample solutions spiked with all the impurities at four different concentration levels of LOQ, $50 \%, 100 \%$ and $250 \%$ of each at the specified limit in both methods. The recovery of all these impurities were found to be in-between the predefined acceptance criterion of $80.0 \%$ $-120.0 \%$

\section{Stability of Analytical Solution}

Rivaroxaban spiked with all impurities at specified level were prepared and analyzed immediately and after different time intervals up to $24 \mathrm{hrs}$ to determine the stability of sample solution in both methods. The sample cooler temperature was maintained at about $25^{\circ} \mathrm{C}$ and at about refrigerator temperature $\left(2-8^{\circ} \mathrm{C}\right)$. The results from these studies indicated that the sample solution was stable at room temperature and at $2-8^{\circ} \mathrm{C}$.

\section{Robustness}

The chromatographic conditions were deliberately altered to evaluate the robustness of developed method. The resolution between closely eluting peaks was evaluated on altered chromatographic condition. To study the effect of flow rate on the resolution, the flow rate of mobile phase was altered by \pm $0.1 \mathrm{~mL} / \mathrm{min}$ (0.9 to $1.1 \mathrm{~mL} / \mathrm{min}$ from $1.0 \mathrm{~mL} / \mathrm{min}$ ). The effect of column temperature on resolution was studied at $40^{\circ} \mathrm{C}$ and $50^{\circ} \mathrm{C}$ instead of $45^{\circ} \mathrm{C}$. whereas all other mobile phase components were held constant similarly to study the $\mathrm{pH}$ effect, $\mathrm{pH}$ of buffer was altered by \pm 0.2 keeping rest parameters same. Buffer: solvent mixture was studied by changing composition $\pm 2 \%$ absolute of solvent mixture. All these parameters were studied by changing one parameter only at a time. The resolution between all known and unknown peaks present in sample was greater than 1.5 in all the deliberate varied chromatographic conditions indicating the robustness of the method.

\section{Conclusion}

A simple accurate and precise HPLC method has been developed for determination of Rivaroxaban and its impurities in bulk drug and dosage form. The method was successfully validated in accordance with ICH guidelines. It can be conveniently used for routine quality control analysis of Rivaroxaban and its impurities in bulk drug and dosage form. Degradation impurities not interference with Rivaroxaban and their impurities, thus the method is stability indicating.

\section{References}

1. Roehrig S, Straub A, Pohlmann J, Lampe T, Pernerstorfer J, Schlemmer $\mathrm{KH}$, et al. Discovery of the novel antithrombotic agent 5-chloro- $\mathrm{N}$ (\{(5S)-2-oxo-3- [4-(3- oxomorpholin-4-yl)phenyl]-1,3-oxazolidin5-yl\}methyl)thiophene- 2-carboxamide (BAY 59-7939): an oral direct factor Xa inhibitor. J Med Chem. 2005;48(19):5900-5908. doi: 10.1021/jm050101d

2. Celebier M, Recber T, Kocak E and Altınoz S. RP-HPLC method development and validation for estimation of rivaroxaban in pharmaceutical dosage forms. Braz J Pharm. 2013;49(2):359-366.

3. Chandrasekhar K, SatyavaniP, Dhanalakshmi A, Devi C, Barik A and Devanaboyina N. A new method development and validation for analysis of rivaroxaban in formulation by RP-HPLC. Research Desk. 2012;1(1): 24-33.

4. Vaghela D, Patel P. High performance thin layer chromatographic method with densitometry analysis for determination of Rivaroxaban from its tablet dosage form. International Journal of Pharmacy and Pharmaceutical Sciences. 2014;6(6):383-386.

5. Jebaliya H, Dabhi B, Patel M, Jadeja Y, Shah A. Stress study and estimation of a potent anticoagulant drug rivaroxaban by a validated HPLC method: Technology transfer to UPLC.Journal of Chemical and Pharmaceutical Research. 2015;7(10):749-765.

6. Pinaz A Kasad, Murali krishna K.S. Design and Validation of Dissolution Profile of Rivaroxaban by Using RP-HPLC Method in Dosage Form. Asian Journal of Pharmaceutical Sciences. 2013; 3(3):75-78. 
7. Rockville M D. General Tests, chapter-621, chromatography system suitability United states pharmacopieial convention (USP), USP 39; 2016.

8. ICH guidelines, validation of analytical procedures test and methodology, Q2(R1). 2003.
9. RafecasJL, ComelyA, FeralliA, Amelia CC, Pasto AM. Process for the preparation of rivaroxaban and intermediates, EP 2521723 A1, 2012. 